\title{
Sets of integers with pairwise common divisor and a factor from a specified set of primes
}

\author{
by
}

\author{
Rudolf Ahlswede and Levon H. Khachatrian (Bielefeld)
}

1. Introduction and main results. Whenever possible we keep the notation of [1], [2]. $\mathbb{N}$ denotes the set of positive integers and $\mathbb{N}^{*}$ is the set of positive squarefree numbers. $\mathbb{P}=\left\{p_{1}, p_{2}, \ldots\right\}=\{2,3,5, \ldots\}$ denotes the set of primes and $p_{s}$ denotes the $s$ th prime.

For two numbers $u, v \in \mathbb{N}$ we write $u \mid v$ (resp. $u \nmid v$ ) iff $u$ divides $v$ (resp. $u$ does not divide $v) ;[u, v]$ stands for the least common multiple of $u$ and $v$; $(u, v)$ is the largest common divisor of $u, v$; and we say that $u$ and $v$ have a common divisor if $(u, v)>1 .\langle u, v\rangle$ denotes the interval $\{x \in \mathbb{N}: u \leq x \leq v\}$ and $(u, v\rangle$ denotes the left-open interval $\{x \in \mathbb{N}: u<x \leq v\}$.

For any set $A \subset \mathbb{N}$ let $A(n)=A \cap\langle 1, n\rangle$ and $|A|$ be the cardinality of $A$. The set of multiples of $A$ is

$$
M(A)=\{m \in \mathbb{N}: a \mid m \text { for some } a \in A\} .
$$

For $u \in \mathbb{N}, u \neq 1, p^{+}(u)$ (resp. $p^{-}(u)$ ) denotes the largest (resp. the smallest) prime factor of $u$.

For any $y \in \mathbb{N}, \pi(y)=|\mathbb{P}(y)|$ denotes the counting function of primes. For any subset of primes $T \subset \mathbb{P}$, and $u \in \mathbb{R}^{+}$we set

$$
\phi(u, T)=\{x \in \mathbb{N}(u):(x, p)=1 \text { for all } p \in T\} .
$$

We note that always $\{1\} \in \phi(u, T)$ for all $T \subset \mathbb{P}, u \geq 1$.

Finally, for a set $A=\left\{a_{1}, \ldots, a_{m}\right\}$ of ordered numbers $a_{1}<\ldots<a_{m}$ we also just write $A=\left\{a_{1}<\ldots<a_{m}\right\}$.

P. Erdős and R. Graham (see [3], [4]) posed the following problem: Let $1<a_{1}<\ldots<a_{k}=n,\left(a_{i}, a_{j}\right) \neq 1$. What is the maximal value of $k$ ? We denote it by $g(n)$.

While in [3] the problem was stated unfortunately with many confusing misprints, in [4] one can find the following conjecture: $g(n)$ equals either $n / p^{-}(n)$ or the number of integers of the form $2 \cdot t, t \leq n / 2,(t, n) \neq 1$. However, it is easy to find a counterexample for this assertion and we 
informed Erdős about this during his visit in Bielefeld in 1992. He then came up with the following formulation: Then

Conjecture 1. Let $n=q_{1}^{\alpha_{1}} \ldots q_{r}^{\alpha_{r}}, \alpha_{i} \geq 1, q_{i} \in \mathbb{P}$, and $q_{1}<\ldots<q_{r}$.

$$
g(n)=\max _{1 \leq j \leq r}\left|M\left(2 q_{1}, \ldots, 2 q_{j}, q_{1} \ldots q_{j}\right) \cap \mathbb{N}(n)\right| .
$$

We consider a more general and seemingly more natural problem: Let $Q=\left\{q_{1}<\ldots<q_{r}\right\} \subset \mathbb{P}$ be any finite set of primes and let $A=\left\{a_{1}<\ldots\right.$ $\left.<a_{k}\right\} \subset \mathbb{N}(n)$ be a set such that for all $1 \leq i, j \leq k$,

$$
\left(a_{i}, a_{j}\right) \neq 1
$$

and

$$
\left(a_{i}, \prod_{i=1}^{r} q_{i}\right)>1 .
$$

Denote by $I(n, Q)$ the set of all such sets. We are interested in the quantity

$$
f(n, Q)=\max \{|A|: A \in I(n, Q)\} .
$$

For special values of $n$, namely $n=q_{1}^{\alpha_{1}} \ldots q_{r}^{\alpha_{r}}$ for some $\alpha_{i} \geq 1$, clearly $a_{f(n, Q)}=n$ and we get exactly the problem of Erdős-Graham.

Our problem can be viewed as being dual to that studied in [1], where a specified set of primes is excluded as factors. Obviously, we can assume that $\{2\} \notin Q$, because otherwise $f(n, Q)=\lfloor n / 2\rfloor$ is realized for the even numbers $\leq n$. Our main result is

Theorem 1. For every finite $Q=\left\{q_{1}<\ldots<q_{r}\right\} \subset \mathbb{P}$ and $n \geq \prod_{i=1}^{r} q_{i}$,

$$
f(n, Q)=\max _{1 \leq j \leq r}\left|M\left(2 q_{1}, \ldots, 2 q_{j}, q_{1} \ldots q_{j}\right) \cap \mathbb{N}(n)\right| .
$$

In particular, Conjecture 1 is true.

We will also show (see Section 6) that the restriction on $n$ in Theorem 1 cannot be ignored.

For given finite $Q=\left\{q_{1}<\ldots<q_{r}\right\} \subset \mathbb{P}$ let us look at our problem in the infinite case, i.e. $A=\left\{a_{1}<a_{2}<\ldots\right\} \subset \mathbb{N}$ satisfies (1.1) and (1.2). What is maximal $\bar{d}_{Q}$ of the asymptotic (upper) density of such $A$ ? Theorem 1 immediately yields

Corollary. For any finite $Q=\left\{q_{1}<\ldots<q_{r}\right\} \subset \mathbb{P}$ we have

$$
\bar{d}_{Q}=\max _{1 \leq j \leq r} \frac{1}{2}\left(1-\prod_{i=1}^{j}\left(1-\frac{1}{q_{i}}\right)+\frac{1}{q_{1} \ldots q_{j}}\right) .
$$

Moreover, this maximum is assumed for a set possessing an asymptotic density. 
It is also natural to formulate the problem for the squarefree case. We define $f^{*}(n, Q)$ as the maximal cardinality of sets $A \subset \mathbb{N}^{*}(n)$ satisfying (1.1) and (1.2).

TheOREM 2. For any finite $Q=\left\{q_{1}<\ldots<q_{r}\right\} \subset \mathbb{P}$ we have

$$
f^{*}(n, Q)=\max _{1 \leq j \leq r}\left|M\left(2 q_{1}, \ldots, 2 q_{j}, q_{1} \ldots q_{j}\right) \cap \mathbb{N}^{*}(n)\right| .
$$

We draw attention to the fact that here we have no restriction on $n$. The proof of Theorem 2 is much easier than that of Theorem 1.

Moreover, Theorem 2 can easily be extended to much more general objects, namely to squarefree quasi-numbers (see [1]).

Sections 2, 3, and 4 provide auxiliary results for the proof of Theorem 1 (and sketch of proof of Theorem 2) in Section 5. We draw particular attention to an auxiliary result in Section 3, which is stated as Theorem 3, because it is of independent interest.

Finally, an example in Section 6 shows that (1.4) does not hold without any condition on $n$. The reader is advised to look first at this example.

2. An auxiliary result for "left compressed sets", "upsets", and "downsets". Let $\mathcal{O}(n, Q)$ denote the set of all optimal sets of $I(n, Q)$, i.e.

$$
\mathcal{O}(n, Q)=\{A \in I(n, Q):|A|=f(n, Q)\}
$$

(see (1.3)). For any $p_{s}, p_{t} \in \mathbb{P}, p_{s}<p_{t}$, we define the "left pushing" operation $L_{s, t}$ on subsets of $\mathbb{N}$. For $B \subset \mathbb{N}$ let

$$
B_{1}=\left\{b \in B: b=b_{1} p_{t}^{\alpha},\left(b_{1}, p_{s} p_{t}\right)=1, \alpha \geq 1,\left(b_{1} p_{s}^{\alpha}\right) \notin B\right\} .
$$

Then

$$
L_{s, t}(B)=\left(B \backslash B_{1}\right) \dot{\cup} B_{2},
$$

where $B_{2}=\left\{c \in \mathbb{N}: c=c_{1} p_{s}^{\beta},\left(c_{1}, p_{s} p_{t}\right)=1, \beta \geq 1,\left(c_{1} p_{t}^{\beta}\right) \in B_{1}\right\}$. Clearly

$$
\left|L_{s, t}(B) \cap \mathbb{N}(n)\right| \geq|B(n)| \quad \text { for every } s, t, s<t \text {, and } n \in \mathbb{N} \text {. }
$$

For $Q \subset \mathbb{P}$ the set $B \subset \mathbb{N}$ is said to be left compressed with respect to $Q$ if

$$
L_{s, t}(B)=B \quad \text { for all } s, t, s<t, p_{t} \in \mathbb{P} \backslash Q
$$

and

$$
L_{s, t}(B)=B \quad \text { for all } s, t, s<t, p_{s}, p_{t} \in Q .
$$

For given $Q \subset \mathbb{P}$, we denote by $\mathcal{C}(Q)$ the set of all subsets of $\mathbb{N}$ which are left compressed with respect to $Q$.

Every finite set $B \subset \mathbb{N}$ can be transformed by finitely many operations $L_{s, t}, s<t$, of the types (2.2) and (2.3) into a member of $\mathcal{C}(Q)$. Since these operations preserve (1.1) and (1.2), we get with (2.1) the following result. 
Lemma 1. For any $Q \subset \mathbb{P}$ and $n \in \mathbb{N}$,

$$
\mathcal{O}(n, Q) \cap \mathcal{C}(Q) \neq \emptyset .
$$

Clearly any $A \in \mathcal{O}(n, Q)$ is an "upset":

$$
A=M(A) \cap \mathbb{N}(n),
$$

and it is also a "downset" in the following sense:

$$
\text { for } a \in A \text { with } a=p_{i_{1}}^{\alpha_{1}} \ldots p_{i_{t}}^{\alpha_{t}} \text { and } \alpha_{i} \geq 1 \text { also } p_{i_{1}} \ldots p_{i_{t}} \in A \text {. }
$$

For every $B \subset \mathbb{N}$ we introduce the unique primitive subset $P(B) \subset B$ which has the properties

$$
b_{1}, b_{2} \in P(B), b_{1} \neq b_{2}, \quad \text { implies } \quad b_{1} \nmid b_{2} \text { and } B \subset M(P(B)) .
$$

We know from (2.5) that for any $A \in \mathcal{O}(n, Q), P(A)$ consists only of squarefree numbers and that by (2.4),

$$
A=M(P(A)) \cap \mathbb{N}(n) .
$$

3. Auxiliary inequalities for sets of numbers with forbidden prime factors. Let $T \subset \mathbb{P}$ with $T=T_{1} \dot{\cup} T_{2}$, where

$$
T_{1} \subset\left\{p_{1}, \ldots, p_{s-1}\right\}, \quad T_{2}=\left\{p_{j_{1}}, \ldots, p_{j_{r}}\right\} ; \quad p_{s}<p_{j_{1}}<\ldots<p_{j_{r}} .
$$

The sets $T_{1}$ and $T_{2}$ can be empty.

LEMma 2. Let $s>1$ and suppose that

$$
r \leq \pi\left(p_{s+\ell-1} p_{s}\right)-s-2 \ell+1 \quad \text { for all } \ell \geq 1 .
$$

Then

$$
2|\phi(u, T)| \leq\left|\phi\left(u p_{s}, T\right)\right| \quad \text { for all } u \in \mathbb{R}^{+} .
$$

Remark 1. A more special form of the lemma was proved (although it was not stated explicitly) in our paper [1]. Actually, in [1] we proved (3.2) for $T_{2}=\emptyset$. In this case we have $r=0$ and the condition (3.1),

$$
0 \leq \pi\left(p_{s+\ell-1} p_{s}\right)-s-2 \ell+1 \quad \text { for all } \ell \geq 1,
$$

always holds. Indeed, since $s>1$ we have $p_{s} \geq 3$ and thus the first inequality in $\pi\left(p_{s+\ell-1} p_{s}\right) \geq \pi\left(3 p_{s+\ell-1}\right) \geq 2 \pi\left(p_{s+\ell-1}\right)$, where the last inequality follows from $\pi(3 x) \geq 2 \pi(x)$, which was shown in [1]. Thus for the quantity in question

$$
\begin{aligned}
\pi\left(p_{s+\ell-1} p_{s}\right)-s-2 \ell+1 & \geq 2 \pi\left(p_{s+\ell-1}\right)-s-2 \ell+1 \\
& =2(s+\ell-1)-s-2 \ell+1=s-1>0 .
\end{aligned}
$$

Proof of Lemma 2. (3.2) is equivalent to

$$
|\phi(u, T)| \leq\left|\phi^{\prime}\left(u p_{s}, T\right)\right|
$$


where $\phi^{\prime}\left(u p_{s}, T\right)=\phi\left(u p_{s}, T\right) \cap\left(u, u p_{s}\right\rangle$. We introduce

$$
\Psi(u, T)=\left\{a \in \phi(u, T): p^{+}(a)<p_{s} \text { or } a=1\right\}
$$

and for $a \in \Psi(u, T)$,

$$
D(a)=\left\{b \in \phi(u, T): b=a d, p^{-}(d) \geq p_{s} \text { or } d=1\right\} .
$$

With these sets we can write $\phi(u, T)$ as a disjoint union

$$
\phi(u, T)=\bigcup_{a \in \Psi(u, T)} D(a) .
$$

Next for $a \in \Psi(u, T)$ we introduce

$$
D^{\prime}(a)=\left\{c \in \phi^{\prime}\left(u p_{s}, T\right): c=a d^{*}, p^{-}\left(d^{*}\right) \geq p_{s}\right\} .
$$

Clearly these sets are disjoint and

$$
\phi^{\prime}\left(u p_{s}, T\right) \supset \bigcup_{a \in \Psi(u, T)} D^{\prime}(a) .
$$

Sufficient for $(3.3)$ is

$$
\left|D^{\prime}(a)\right| \geq|D(a)| \quad \text { for all } a \in \Psi(u, T) .
$$

From the definition of $D(a)$ and $D^{\prime}(a)$ it follows that for $T^{*}=\left\{p_{1}, \ldots, p_{s-1}\right\}$ $\cup T_{2}$ we have $|D(a)|=\phi\left(u / a, T^{*}\right),\left|D^{\prime}(a)\right|=\mid \phi^{\prime}\left(u p_{s} / a, T^{*}\right)$, and

$$
\phi^{\prime}\left(u p_{s} / a, T^{*}\right)=\phi\left(u p_{s} / a, T^{*}\right) \backslash \phi\left(u / a, T^{*}\right)=\phi\left(u p_{s} / a, T^{*}\right) \cap\left(u / a, u p_{s} / a\right\rangle .
$$

Thus we arrived at the following sufficient condition for (3.4):

(3.5) $\left|\phi\left(v, T^{*}\right)\right| \leq\left|\phi^{\prime}\left(v p_{s}, T^{*}\right)\right|=\left|\phi\left(v p_{s}, T^{*}\right) \backslash \phi\left(v, T^{*}\right)\right| \quad$ for all $v \in \mathbb{R}^{+}$.

We avoid the trivial cases $v<1$, for which $\phi\left(v, T^{*}\right)=\emptyset$, and $1 \leq v<p_{s}$, for which $\left|\phi\left(v, T^{*}\right)\right|=1$ and $p_{s} \in \phi^{\prime}\left(v p_{s}, T^{*}\right)$. Hence we assume $v \geq p_{s}$ and introduce

$$
F\left(v, T^{*}\right)=\left\{b \in \phi\left(v, T^{*}\right), b \neq 1: b p^{+}(b) \leq v\right\} \cup\{1\} .
$$

Then $\phi\left(v, T^{*}\right)$ is a disjoint union

$$
\phi\left(v, T^{*}\right)=\bigcup_{b \in F\left(v, T^{*}\right)} \tau(b) \cup\{1\},
$$

where $\tau(b)=\left\{m \in \mathbb{N}: m=p b ; p \in \mathbb{P} \backslash T^{*} ; p^{+}(b) \leq p \leq v / b\right\}$. Hence for all $b \in F\left(v, T^{*}\right)$,

$$
|\tau(b)|=\left|\left\{p \in \mathbb{P} \backslash T^{*}: p^{+}(b) \leq p \leq v / b\right\}\right|
$$

and

$$
\left|\phi\left(v, T^{*}\right)\right|=\sum_{b \in F\left(v, T^{*}\right)}|\tau(b)|+1,
$$

where the 1 accounts for the element $\{1\} \in \phi\left(v, T^{*}\right)$. 
On the other hand, we have

$$
\phi^{\prime}\left(v p_{s}, T^{*}\right) \supset \bigcup_{b \in F\left(v, T^{*}\right)} \tau_{1}(b) \cup\left\{p_{s}^{k}\right\},
$$

where $\tau_{1}(b)=\left\{m_{1} \in \mathbb{N}: m_{1}=p b, p \in \mathbb{P} \backslash T^{*}, v / b<p \leq v p_{s} / b\right\}$ and $p_{s}^{k}$ satisfies $v<p_{s}^{k} \leq v p_{s}$ for some $k \in \mathbb{N}$.

It is easy to see that the sets $\left\{\tau_{1}(b)\right\}, b \in F\left(v, T^{*}\right)$, are disjoint and that the element $\left\{p_{s}^{k}\right\}$ does not belong to any of them.

We have

$$
\left|\tau_{1}(b)\right|=\left|\left\{p \in \mathbb{P} \backslash T^{*}: v / b<p \leq v p_{s} / b\right\}\right|
$$

for all $b \in F\left(v, T^{*}\right)$ and

$$
\left|\phi^{\prime}\left(v p_{s}, T^{*}\right)\right| \geq \sum_{b \in F\left(v, T^{*}\right)}\left|\tau_{1}(b)\right|+1,
$$

where the 1 accounts for $\left\{p_{s}^{k}\right\}$. From (3.7) and (3.9) it follows that sufficient for $(3.5)$ is

$$
\left|\tau_{1}(b)\right| \geq|\tau(b)| \quad \text { for all } b \in F\left(v, T^{*}\right) .
$$
have

Let $p_{s+\ell-1} \leq v / b<p_{s+\ell}$ for some $\ell \geq 1$. Then, from (3.6) and (3.8), we

$$
|\tau(b)|=\left|\left\{p \in \mathbb{P} \backslash T^{*}: p^{+}(b) \leq p \leq v / b\right\}\right| \leq\left|\left\{p \in \mathbb{P}: p_{s} \leq p \leq p_{s+\ell-1}\right\}\right|=\ell
$$

and

$$
\begin{aligned}
\left|\tau_{1}(b)\right| & =\left|\left\{p \in \mathbb{P} \backslash T^{*}: v / b<p \leq v p_{s} / b\right\}\right| \\
& \geq\left|\left\{p \in \mathbb{P} \backslash T^{*}: p_{s+\ell-1}<p \leq p_{s+\ell-1} p_{s}\right\}\right| \\
& =\pi\left(p_{s+\ell-1} p_{s}\right)-(s+\ell-1)-r_{1},
\end{aligned}
$$

where $r_{1}$ is the number of primes from $T_{2}$ in the interval $\left\langle p_{s+\ell}, p_{s+\ell-1} p_{s}\right\rangle$. Since $r_{1} \leq r=\left|T_{2}\right|$ we have

$$
\left|\tau_{1}(b)\right| \geq \pi\left(p_{s+\ell-1} p_{s}\right)-(s+\ell-1)-r .
$$

Finally, using condition (3.1) we have established the sufficient condition

$$
\left|\tau_{1}(b)\right| \geq \pi\left(p_{s+\ell-1} p_{s}\right)-(s+\ell-1)-r \geq \ell \geq|\tau(b)| .
$$

Re mark 2. Perhaps one can try to simplify condition (3.1) in Lemma 2 by finding $\min _{\ell \in \mathbb{N}}\left(\pi\left(p_{s+\ell-1} p_{s}\right)-2 \ell\right)$ for $s \geq 2$. However, if the minimum is achieved for $\ell=1$ (which seems most likely), then one has at least to prove that between $p_{s}^{2}$ and $p_{s} p_{s+1}$ there are at least two primes, which seems hopeless. For comparison let us recall that in 1904 Brocard conjectured that between $p_{s}^{2}$ and $p_{s+1}^{2}$, there are at least 4 primes and this remains unsolved (see $[5])$. 
We need the following result, which is probably known to the experts (in fact, it is an easy consequence of known results), but we were not able to find it in the literature.

Lemma 3. $p_{s} p_{t}>p_{\text {st }}$ for all $s, t \in \mathbb{N}$ except two cases, namely, $s=3$, $t=4$, for which $p_{3} p_{4}=5 \cdot 7=35<p_{12}=37$, and $s=t=4$, for which $p_{4} p_{4}=7 \cdot 7=49<p_{16}=53$.

P r o of. We use very sharp estimates of the size of primes, due to Rosser and Schoenfeld [6]:

$$
\begin{aligned}
& p_{n}<n(\log n+\log \log n-1 / 2) \quad \text { for } n \geq 20, \\
& p_{n}>n \log n \quad \text { for } n \geq 1 .
\end{aligned}
$$

Using (3.10) one gets $p_{s} p_{t}>p_{s t}$ for all $t \geq s \geq 12$. For every $s \leq 11$, we take the exact value of $p_{s}$ and estimate, using (3.10), only primes $p_{t}$ and $p_{s t}$. For example, let $s=4, p_{4}=7, t \geq 5$. Since $s t \geq 20$ we can use (3.10) to get

$$
p_{4 t}<4 t(\log 4 t+\log \log 4 t-1 / 2) \quad \text { and } \quad p_{4} p_{t}=7 p_{t}>7 \cdot t \log t .
$$

From (3.11) we have $7 p_{t}>p_{4 t}$ for all $t \geq 25$ and the cases $5 \leq t \leq 24$ are verified by inspection using the list of primes. In the case $s=t=4$ we have the opposite inequality and this is one of the two exceptions specified in the lemma. For other values of $s \leq 11$ we have similar calculations.

We recall the definitions of the sets $T_{1}, T_{2}, T$ in Lemma 2 :

$$
T_{1} \subset\left\{p_{1}, \ldots, p_{s-1}\right\}, \quad T_{2}=\left\{p_{j_{1}}, \ldots, p_{j_{r}}\right\} ; \quad p_{s}<p_{j_{1}}<\ldots<p_{j_{r}} ;
$$

and $s>1$. We introduce

$$
T_{3}=\left(\left\{p_{1}, \ldots, p_{s-1}\right\} \backslash T_{1}\right) \cup\left\{p_{s}\right\}=\left\{p_{i_{1}}, \ldots, p_{i_{t}}\right\}, \quad p_{i_{1}}<\ldots<p_{i_{t}}=p_{s} .
$$

TheOREM 3. Let $s>1$ and consider the sets $T_{1}, T_{2}, T_{3}, T=T_{1} \cup T_{2}$ of primes as described above. Then for every $u \in \mathbb{R}^{+}$with

$$
u \geq \frac{\prod_{p \in T_{2}} p}{\prod_{p \in T_{3}} p},
$$

we have

$$
2|\phi(u, T)| \leq\left|\phi\left(u p_{s}, T\right)\right| .
$$

P r o of. In the light of Lemma 2 we can assume

$$
r>\pi\left(p_{s+\ell-1} p_{s}\right)-s-2 \ell+1 \quad \text { for some } \ell \geq 1 .
$$

First let us show that from (3.14) one can get

$$
r>(s-1)^{2} .
$$

Indeed, from Lemma 3 we know that $p_{s+\ell-1} p_{s}>p_{(s+\ell-1) s}$ for all $s, \ell$ except $s=3, \ell=2$ and $s=4, \ell=1$. 
Hence

$$
\pi\left(p_{s+\ell-1} p_{s}\right) \geq \pi\left(p_{(s+\ell-1) s}\right)=s(s+\ell-1)
$$

for all $s, \ell$ with the exceptions mentioned above. Therefore

$$
r>\pi\left(p_{s+\ell-1} p_{s}\right)-s-2 \ell+1 \geq s(s+\ell-1)-s-2 \ell+1 \geq(s-1)^{2}
$$

since $s>1$. For $s=3, \ell=2$ and $s=4, \ell=1$ we verify (3.15) by inspection.

Now, for every $u \in \mathbb{R}^{+}$by the inclusion-exclusion principle we have

$$
|\phi(u, T)|=\lfloor u\rfloor-\sum_{p \in T}\left\lfloor\frac{u}{p}\right\rfloor+\sum_{\substack{p<q \\ p, q \in T}}\left\lfloor\frac{u}{p q}\right\rfloor \ldots \leq u \prod_{p \in T}\left(1-\frac{1}{p}\right)+2^{|T|-1}
$$

and

$$
\left|\phi\left(u p_{s}, T\right)\right| \geq u p_{s} \prod_{p \in T}\left(1-\frac{1}{p}\right)-2^{|T|-1} .
$$

Hence, sufficient for (3.13) is

$$
u\left(p_{s}-2\right) \prod_{p \in T}\left(1-\frac{1}{p}\right) \geq 3 \cdot 2^{|T|-1} \quad \text { for all } u \geq \frac{\prod_{p \in T_{2}} p}{\prod_{p \in T_{3}} p} .
$$

Since $|T|=s-t+r,(3.16)$ is equivalent to

$$
\begin{aligned}
& \left(p_{s}-2\right) \frac{\prod_{p \in T_{2}} p}{\prod_{p \in T_{3}} p} \prod_{p \in T_{1} \cup T_{2}}\left(1-\frac{1}{p}\right) \\
& =\left(p_{s}-2\right) \frac{\prod_{p \in T_{2}}(p-1)}{\prod_{i=1}^{s} p_{i}} \prod_{p \in T_{1}}(p-1) \geq 3 \cdot 2^{r} \cdot 2^{s-t-1} .
\end{aligned}
$$

Since $\left|T_{1}\right|=s-t$, we observe that

$$
\prod_{p \in T_{1}}(p-1) \geq 2^{s-t-1}
$$

and sufficient for $(3.17)$ is

$$
\left(p_{s}-2\right) \frac{\prod_{p \in T_{2}}(p-1)}{\prod_{i=1}^{s} p_{i}} \geq 3 \cdot 2^{r} .
$$

Now, if $s \geq 3$, then

$$
\begin{aligned}
\left(p_{s}-2\right) \frac{\prod_{p \in T_{2}}(p-1)}{\prod_{i=1}^{s} p_{i}} & =\left(p_{s}-2\right) \frac{\left(p_{j_{1}}-1\right) \ldots\left(p_{j_{r}}-1\right)}{p_{1} \ldots p_{s}} \\
& >\left(p_{s}-2\right)\left(p_{j_{s+1}}-1\right) \ldots\left(p_{j_{r}}-1\right) \\
& >\left(p_{s}-2\right)\left(p_{j_{s+1}}-1\right)^{r-s} \geq\left(p_{s}-2\right) \cdot 16^{r-s}>3 \cdot 2^{r},
\end{aligned}
$$

since $p_{j_{1}} \geq 7, p_{j_{s+1}} \geq 17$ and we know that $r \geq(s-1)^{2}+1(\operatorname{see}(3.15))$. 
So, it remains to show the validity of (3.13) only for the case $s=2$. From (3.15) we know that $r \geq 2$ and, if $r>2$, we have in (3.18)

$$
\begin{aligned}
& \frac{\left(p_{j_{1}}-1\right)\left(p_{j_{2}}-1\right) \ldots\left(p_{j_{r}}-1\right)}{2 \cdot 3} \\
& \quad \geq \frac{(5-1)(7-1)(11-1) \ldots\left(p_{j_{r}}-1\right)}{6} \geq 3 \cdot 2^{r} .
\end{aligned}
$$

Hence, we can assume $r=2$. However, the formula (3.18) does not hold in this case for instance for $p_{j_{1}}=5, p_{j_{2}}=7$ :

$$
\frac{(5-1)(7-1)}{6} \nsucceq 3 \cdot 2^{2}=12 \text {. }
$$

In the case $s=r=2$ we have to estimate the quantities $|\phi(u, T)|$ and $|\phi(3 u, T)|$ more accurately.

We have to consider two cases: $t=1$ and $t=2$, where $t=\left|T_{3}\right|$. We are going to prove (3.13) only for $t=1$ (the case $t=2$ is similar, actually even simpler). We have to prove that for $q_{1}, q_{2}$ with $5 \leq q_{1}<q_{2}$ and $T=$ $\left\{2, q_{1}, q_{2}\right\}, 2|\phi(u, T)|<|\phi(3 u, T)|$ holds provided that $u \geq q_{1} q_{2} / 3$. We have

$$
\begin{aligned}
&|\phi(3 u, T)|-2|\phi(u, T)| \\
&=\lfloor 3 u\rfloor-\left\lfloor\frac{3 u}{2}\right\rfloor-\left\lfloor\frac{3 u}{q_{1}}\right\rfloor-\left\lfloor\frac{3 u}{q_{2}}\right\rfloor+\left\lfloor\frac{3 u}{2 q_{1}}\right\rfloor \\
&+\left\lfloor\frac{3 u}{2 q_{2}}\right\rfloor+\left\lfloor\frac{3 u}{q_{1} q_{2}}\right\rfloor-\left\lfloor\frac{3 u}{2 q_{1} q_{2}}\right\rfloor-2\lfloor u\rfloor+2\left\lfloor\frac{u}{2}\right\rfloor+2\left\lfloor\frac{u}{q_{1}}\right\rfloor+2\left\lfloor\frac{u}{q_{2}}\right\rfloor \\
&-2\left\lfloor\frac{u}{2 q_{1}}\right\rfloor-2\left\lfloor\frac{u}{2 q_{2}}\right\rfloor-2\left\lfloor\frac{u}{q_{1} q_{2}}\right\rfloor+2\left\lfloor\frac{u}{2 q_{1} q_{2}}\right\rfloor \\
&=\left(\lfloor 3 u\rfloor-\left\lfloor\frac{3 u}{2}\right\rfloor-2\lfloor u\rfloor+2\left\lfloor\frac{u}{2}\right\rfloor\right) \\
&-\left(\left\lfloor\frac{3 u}{q_{1}}\right\rfloor-\left\lfloor\frac{3 u}{2 q_{1}}\right\rfloor-2\left\lfloor\frac{u}{q_{1}}\right\rfloor+2\left\lfloor\frac{u}{2 q_{1}}\right\rfloor\right) \\
&-\left(\left\lfloor\frac{3 u}{q_{2}}\right\rfloor-\left\lfloor\frac{3 u}{2 q_{2}}\right\rfloor-2\left\lfloor\frac{u}{q_{2}}\right\rfloor+2\left\lfloor\frac{u}{2 q_{2}}\right\rfloor\right) \\
&+\left(\left\lfloor\frac{3 u}{q_{1} q_{2}}\right\rfloor-\left\lfloor\frac{3 u}{2 q_{1} q_{2}}\right\rfloor-2\left\lfloor\frac{u}{q_{1} q_{2}}\right\rfloor+2\left\lfloor\frac{u}{2 q_{1} q_{2}}\right\rfloor\right) .
\end{aligned}
$$

Now we use the following inequalities (which can be easily verified):

$$
\begin{aligned}
x-1 & <x-\frac{5}{6}<\lfloor 6 x\rfloor-\lfloor 3 x\rfloor-2\lfloor 2 x\rfloor+2\lfloor x\rfloor \\
& \leq x+\frac{5}{6}<x+1 \quad \text { for all } x \in \mathbb{R}^{+}
\end{aligned}
$$


to get

$$
\begin{aligned}
|\phi(3 u, T)|-2|\phi(u, T)| & >u\left(1-\frac{1}{2}\right)\left(1-\frac{1}{q_{1}}\right)\left(1-\frac{1}{q_{2}}\right)-4 \\
& =\frac{u\left(q_{1}-1\right)\left(q_{2}-1\right)}{2 q_{1} q_{2}}-4 \geq \frac{\left(q_{1}-1\right)\left(q_{2}-1\right)}{6}-4 \geq 0,
\end{aligned}
$$

since $u \geq q_{1} q_{2} / 3$ and $5 \leq q_{1}<q_{2}$.

Remarks. 3. We note that (3.13) does not always hold if we ignore the restriction on $u$. For example, for $T=\{2,5,7\}, s=2, u=3$ we have

$2|\phi(3, T)|=2|\{1,3\}|=4 \not \leq\left|\phi\left(p_{2} \cdot 3, T\right)\right|=|\phi(9, T)|=|\{1,3,9\}|=3$.

4. If $u$ is sufficiently large, $u>u(\varepsilon)$, then the coefficient 2 in (3.13) of the theorem (in Lemma 2 as well) clearly can be changed to $p_{s}-\varepsilon$, for any $\varepsilon>0$.

4. Further preparations: Lemmas 4, 5, 6. For given $Q \subset \mathbb{P}$ and any $b \in \mathbb{N}$ let $p^{+}(b, Q)$ denote the maximal prime from $\mathbb{P} \backslash Q$ which occurs in the prime decomposition of $b$ (in the case $Q=\emptyset$ we always have $p^{+}(b, \phi)=$ $\left.p^{+}(b)\right)$. If $b$ is completely composed of primes from $Q$ or $b=1$, then $p^{+}(b, Q)=1$. Further, let $A \subset \mathbb{N}(n)$ be such that $P(A)$, the primitive subset of $A$, consists only of squarefree numbers and let $A=M(P(A)) \cap \mathbb{N}(n)$. For given $Q \subset \mathbb{P}$, we define

$$
p^{+}(P(A), Q)=\max _{a \in P(A)} p^{+}(a, Q) .
$$

We consider $L_{i, j}(A)=A^{\prime}$, where $i<j$ and $p_{j} \in Q$ implies $p_{i} \in Q$. One can easily verify the following statement.

Lemma $4 . p^{+}(P(A), Q) \geq p^{+}\left(P\left(A^{\prime}\right), Q\right)$.

Let $A \in \mathcal{O}(n, Q) \cap C(Q)$ for some $Q=\left\{q_{1}, \ldots, q_{r}\right\}, 2<q_{1}<\ldots<q_{r}$ and $n \in \mathbb{N}$. We know (see Lemma 1 ) that such a set $A$ always exists. Let $P(A)$ be the primitive subset of $A$ and $p^{+}(P(A), Q)=p_{s}$ for some $p_{s} \in(\mathbb{P} \backslash Q) \cup\{1\}$. We write $P(A)$ in the form $P(A)=R_{0} \dot{\cup} R_{1} \dot{\cup} \ldots \dot{\cup} R_{s}$, where

$$
R_{0}=\left\{a \in P(A): p^{+}(a, Q)=1\right\}
$$

and

$$
R_{i}=\left\{a \in P(A): p^{+}(a, Q)=p_{i}\right\}, \quad 1 \leq i \leq s .
$$

We note that some of the $R_{i}$ can be empty, but not $R_{s}$.

Since $A$ is optimal, we know that $A=M(P(A)) \cap \mathbb{N}(n)$, which can be written in the form

$$
A=M(P(A)) \cap \mathbb{N}(n)=\left(M\left(R_{0} \dot{\cup} \ldots \dot{\cup} R_{s-1}\right) \dot{\cup} K\left(R_{s}\right)\right) \cap \mathbb{N}(n),
$$


where $K\left(R_{s}\right)=\left(M\left(R_{s}\right) \backslash M\left(R_{0} \cup \ldots \cup R_{s-1}\right)\right) \cap \mathbb{N}(n)$, i.e. $K\left(R_{s}\right)$ is the set of those elements of $A$ which are not divisible by any $b \in R_{0} \cup \ldots \cup R_{s-1}$.

Let $s>1$ and $R_{s}=R_{s}^{0} \dot{\cup} R_{s}^{1}$, where

$$
R_{s}^{0}=\left\{b \in R_{s}: 2 \mid b\right\}, \quad R_{s}^{1}=R_{s} \backslash R_{s}^{0},
$$

and $K\left(R_{s}\right)=K^{0}\left(R_{s}\right) \dot{\cup} K^{1}\left(R_{s}\right)$, where

$$
K^{0}\left(R_{s}\right)=\left\{a \in K\left(R_{s}\right): 2 \mid a\right\}, \quad K^{1}\left(R_{s}\right)=K\left(R_{s}\right) \backslash K^{0}\left(R_{s}\right) .
$$

Finally, let

$$
G_{s}^{i}=\left\{m \in \mathbb{N}: m p_{s} \in R_{s}^{i}\right\}, \quad i=0,1 .
$$

Lemma 5. Let $A \in \mathcal{O}(n, Q) \cap C(Q)$, let the sets $K^{i}\left(R_{s}\right), R_{s}^{i}, G_{s}^{i}, i=0,1$, be defined as above, and let $s>1$. Then

(1) $b \nmid a$ for all $b \in R_{s}^{i}, a \in K^{1-i}\left(R_{s}\right), i=0,1$,

(2) $K^{i}\left(R_{s}\right)=M\left(R_{s}^{i}\right) \backslash M\left(R_{0} \cup \ldots \cup R_{s-1}\right), i=0,1$,

(3) $G_{s}^{i} \in I(n, Q), i=0,1$ (defined in the introduction),

(4) $\left(R_{0} \cup \ldots \cup R_{s-1} \cup G_{s}^{i}\right) \in I(n, Q), i=0,1$.

Proof. (1) Obviously, $b \nmid a$ for all $b \in R_{s}^{0}$ and $a \in K^{1}\left(R_{s}\right)$. Suppose $b \mid a$ for some $b \in R_{s}^{1}, a \in K^{0}\left(R_{s}\right)$. Then $\frac{b}{p_{s}} \cdot 2 \mid a$ as well, because $2 \nmid b$ and $2 \mid a$. However $\frac{b}{p_{s}} \cdot 2 \in A$, because $A$ is left compressed with respect to $Q$ and $p_{s} \notin Q, p_{s}>2,2 \nmid b$. Hence $\frac{b}{p_{s}} \cdot 2 \in M\left(R_{0} \cup \ldots \cup R_{s-1}\right)$, because $p_{s} \nmid \frac{b}{p_{s}} \cdot 2$. Therefore $a \notin K\left(R_{s}\right)$, becuase $\frac{b}{p_{s}} \cdot 2 \mid a$. This is a contradiction.

(2) follows from (1).

(3) Clearly $G_{s}^{0} \in I(n, Q)$, because all elements of $G_{s}^{0}$ are even and $p_{s} \notin Q$. Let us show that $G_{s}^{1} \in I(n, Q)$ as well. Suppose to the contrary that there exist $b_{1}, b_{2} \in G_{s}^{1}$ with $\left(b_{1}, b_{2}\right)=1$. We have $b_{1} p_{s}, b_{2} p_{s} \in R_{s}^{1}$ (see definition of $G_{s}^{1}$ and $R_{s}^{1}$ ). However, since $R_{s}^{1} \subset A, A$ is left compressed with respect to $Q$ and $p_{s} \notin Q, p_{s}>2,2 \nmid b_{1}, 2 \nmid b_{2}$, we conclude $2 b_{1} \in A$ as well. Hence $2 b_{1}$, $p_{s} b_{2} \in A$ and at the same time $\left(2 b_{1}, p_{s} b_{2}\right)=1$, which is a contradiction.

(4) This is trivial.

Finally, we need an auxiliary result concerning the set $K\left(R_{s}\right)$. Let $a$ be any element of $K\left(R_{s}\right)$. It can be uniquely written in the form

$$
a=p_{i_{1}}^{\alpha_{1}} \ldots p_{i_{t}}^{\alpha_{t}} \cdot q_{j_{1}}^{\beta_{1}} \ldots q_{j_{\ell}}^{\beta_{\ell}} a_{3},
$$

where $p_{i_{1}}<\ldots<p_{i_{t}}=p_{s}<q_{j_{1}}<\ldots<q_{j_{\ell}} ; \alpha_{i}, \beta_{i} \geq 1, q_{j_{i}} \in Q, p^{-}\left(a_{3}\right)>$ $p_{s}, p \mid a_{3}$ implies $p \in \mathbb{P} \backslash Q$ or $a_{3}=1$. We note that $\left\{q_{j_{1}}, \ldots, q_{j_{\ell}}\right\}=\emptyset$ is also possible.

Lemma 6. Let $A \in \mathcal{O}(n, Q) \cap C(Q)$ with $p^{+}(p(A), Q)=p_{s}, s>1$, and let $a \in K\left(R_{s}\right)$ be an element of the form (4.6). Then 
(1) $a^{\prime}=p_{i_{1}}^{\alpha_{1}^{\prime}} \ldots p_{i_{t}}^{\alpha_{t}^{\prime}} \cdot q_{j_{1}}^{\beta_{1}^{\prime}} \ldots q_{j_{\ell}}^{\beta_{\ell}^{\prime}} a_{3}^{\prime} \in K\left(R_{s}\right)$ if $a^{\prime} \leq n, \alpha_{i}^{\prime} \geq 1, \beta_{i}^{\prime} \geq 1$ and $a_{3}^{\prime}$ either equals 1 or satisfies $p^{-}\left(a_{3}^{\prime}\right)>p_{s}$ and $p \mid a_{3}^{\prime}$ implies $p \in \mathbb{P} \backslash Q$.

(2) For every integer $b \in \mathbb{N}$ of the form $b=p_{i_{1}}^{\gamma_{1}} \ldots p_{i_{t-1}}^{\gamma_{t-1}} \cdot q_{j_{1}}^{\delta_{1}} \ldots q_{j_{\ell}}^{\delta_{\ell}} \cdot b^{\prime}$ we have $b \notin A$ if $\gamma_{i} \geq 0, \delta_{i} \geq 0$ and $b^{\prime}$ either equals 1 or satisfies $p^{-}\left(b^{\prime}\right)>p_{s}$ and $p \mid b^{\prime}$ implies $p \in \mathbb{P} \backslash Q$.

Proof. (1) Since $a \in K\left(R_{s}\right) \subset A$, we have $m \mid a$ for some $m \in P(A)$ and hence $m \mid p_{i_{1}} \ldots p_{i_{t}} \cdot q_{j_{1}} \ldots q_{j_{\ell}}$, because $p^{+}(P(A), Q)=p_{s}$ and $m \in P(A)$ implies $m \in \mathbb{N}^{*}$. Therefore all integers of the form in (1) belong to our set $A$. However, every $m \in P(A)$ with $m \mid a$ must belong to $R_{s}$, otherwise $a \notin K\left(R_{s}\right)$, and this completes the proof of (1).

(2) If for some $b \in \mathbb{N}$ of the form in (2) we have $b \in A$, then $m^{\prime} \mid b$ for some $m^{\prime} \in R_{0} \cup \ldots \cup R_{s-1}\left(m^{\prime} \notin R_{s}\right.$, because $\left.p_{s} \nmid b\right)$. Since $A$ is a "downset", $p^{+}\left(R_{0} \cup \ldots \cup R_{s-1}, Q\right) \leq s-1$ and since $p^{-}\left(b^{\prime}\right)>p_{s}, p \mid b^{\prime}$ implies $p \in \mathbb{P} \backslash Q$ or $b^{\prime}=1$, we have $m^{\prime} \mid p_{i_{1}} \ldots p_{i_{t-1}} \cdot q_{j_{1}} \ldots q_{j_{\ell}}$ as well, and hence $m^{\prime} \mid a$, which is a contradiction to $a \in K\left(R_{s}\right)$.

Let

$$
\begin{array}{r}
Z=\left\{a^{*} \in K\left(R_{s}\right) \cap \mathbb{N}^{*}: a^{*}=p_{i_{1}} \ldots p_{i_{t}} \cdot q_{j_{1}} \ldots q_{j_{\ell}}, p_{i_{1}}<\ldots<p_{i_{t}}\right. \\
\left.=p_{s}<q_{j_{1}}<\ldots<q_{j_{\ell}}, q_{j_{i}} \in Q\right\}
\end{array}
$$

and for $a^{*} \in Z$, let $E\left(a^{*}\right)$ denote the set of all integers $a^{\prime}$ of the form (1) in Lemma 6 with $a^{\prime} \leq n$. Lemma 6(1) immediately yields

$$
K\left(R_{s}\right)=\bigcup_{a^{*} \in Z} E\left(a^{*}\right) .
$$

Finally, from Lemma 5(1) and (4.7) we have

$$
K^{i}\left(R_{s}\right)=\bigcup_{a^{*} \in Z^{i}} E\left(a^{*}\right), \quad i=0,1,
$$

where $Z^{i}=Z \cap K^{i}\left(R_{s}\right), i=0,1$.

5. Proof of Theorem 1. Let $Q=\left\{q_{1}, \ldots, q_{r}\right\}, 2<q_{1}<\ldots<q_{r}$, $n \in \mathbb{N}, n \geq \prod_{i=1}^{r} q_{i}$ and let $\mathcal{O}(n, Q)$ be the set of all optimal sets. For every $B \in \mathcal{O}(n, Q)$ we consider $P(B)$ the primitive, generating subset of $B: B=M(P(B)) \cap \mathbb{N}(n)$.

Let

$$
p_{s}=\min _{B \in \mathcal{O}(n, Q)} p^{+}(P(B), Q),
$$

where $p^{+}(P(B), Q)$ is defined in (4.1), and $p_{s} \in(\mathbb{P} \backslash Q) \cup\{1\}$. Let $\mathcal{O}_{1}(n, Q)=$ $\left\{B \in \mathcal{O}(n, Q): p^{+}(P(B), Q)=p_{s}\right\}$. Our first step is to prove

$$
p_{s} \leq 2 \text {. }
$$

From Lemmas 1 and 4 it follows that $\mathcal{O}_{1}(n, Q) \cap C(Q) \neq \emptyset$. 
Let $A \in \mathcal{O}_{1}(n, Q) \cap C(Q)$ and suppose, contrary to (5.1), that $p_{s} \geq 3$, i.e. $s \geq 2$. Let $P(A)=R_{0} \cup R_{1} \cup \ldots \cup R_{s}$, where the $R_{i}$ 's are described in (4.2). We also use the sets $R_{s}^{i}, G_{s}^{i}, K^{i}\left(R_{s}\right)$ (see (4.3), (4.4)). We consider the following two sets:

$$
A^{i}=M\left(R_{0} \cup \ldots \cup R_{s-1} \cup G_{s}^{i}\right) \cap \mathbb{N}(n), \quad i=0,1 .
$$

From Lemma 5 we know that $A^{0}, A^{1} \in I(n, Q)$ and we are going to prove that at least one of the inequalities $\left|A^{0}\right| \geq|A|$ or $\left|A^{1}\right| \geq|A|$ holds. Suppose

$$
\left|K^{1}\left(R_{s}\right) \cap \mathbb{N}(n)\right| \geq\left|K^{0}\left(R_{s}\right) \cap \mathbb{N}(n)\right|
$$

(the opposite case is symmetrically the same), and let us prove that

$$
\left|A^{1}\right|=\left|M\left(R_{0} \cup \ldots \cup R_{s-1} \cup G_{s}^{1}\right) \cap \mathbb{N}(n)\right| \geq|A| .
$$

Let

$$
K^{*}=\left(M\left(G_{s}^{1}\right) \backslash M\left(R_{0} \cup \ldots \cup R_{s-1}\right)\right) \cap \mathbb{N}(n) .
$$

In the light of (5.2), sufficient for (5.3) is

$$
\left|K^{*}\right| \geq 2\left|K^{1}\left(R_{s}\right)\right| \text {. }
$$

From (4.8) we know that

$$
K^{1}\left(R_{s}\right)=\bigcup_{a^{*} \in Z^{1}} E\left(a^{*}\right)
$$

where

$$
\begin{array}{r}
Z^{1}=\left\{a^{*} \in K^{1}\left(R_{s}\right) \cap \mathbb{N}^{*}: a^{*}=p_{i_{1}} \ldots p_{i_{t}} \cdot q_{j_{1}} \ldots q_{j_{\ell}}, p_{j_{1}}<\ldots<p_{i_{t}}\right. \\
\left.=p_{s}<q_{j_{1}}<\ldots<q_{j_{\ell}}, q_{j_{i}} \in Q\right\}
\end{array}
$$

and

$$
\begin{aligned}
E\left(a^{*}\right)= & \left\{a \leq n: a=p_{i_{1}}^{\alpha_{1}} \ldots p_{i_{t}}^{\alpha_{t}} \cdot q_{j_{1}}^{\beta_{1}} \ldots q_{j_{\ell}}^{\beta_{\ell}} \cdot a_{3}, \alpha_{i} \geq 1, \beta_{i} \geq 1, p^{-}\left(a_{3}\right)>p_{s},\right. \\
& \left.p \mid a_{3} \Rightarrow p \in \mathbb{P} \backslash Q \text { or } a_{3}=1, \text { and } a^{*}=p_{i_{1}} \ldots p_{i_{t}} \cdot q_{j_{1}} \ldots q_{j_{\ell}} \in Z^{1}\right\} .
\end{aligned}
$$

It is easy to see that one can write

$$
E\left(a^{*}\right)=\left\{a \leq n: a=a^{*} \cdot a_{3}^{\prime}, a_{3}^{\prime} \in \phi\left(n / a^{*}, T\right)\right\},
$$

where $T=\left(\left\{p_{1}, \ldots, p_{s}\right\} \backslash\left\{p_{i_{1}}, \ldots, p_{i_{t}}\right\}\right) \cup\left(\left\{q \in Q: q>p_{s}\right\} \backslash\left\{q_{j_{1}}, \ldots, q_{j_{\ell}}\right\}\right)$. Hence

$$
\left|E\left(a^{*}\right)\right|=\left|\phi\left(n / a^{*}, T\right)\right|
$$

for every $a^{*} \in Z^{1}$ and $T=T\left(a^{*}\right)$, as described in (5.6).

Now, for any $a^{*} \in Z^{1}, a^{*}=p_{i_{1}} \ldots p_{i_{t}} \cdot q_{j_{1}} \ldots q_{j_{\ell}}, p_{i_{1}}<\ldots<p_{i_{t}}=$ $p_{s}<q_{j_{1}}<\ldots<q_{j_{\ell}}, q_{j_{i}} \in Q$, we consider the integer $b^{*}=a^{*} / p_{s}=$ $p_{i_{1}} \ldots p_{i_{t-1}} \cdot q_{j_{1}} \ldots q_{j_{\ell}}$ and the set

$$
\begin{gathered}
E^{*}\left(b^{*}\right)=\left\{b \leq n: b=p_{i_{1}}^{\gamma_{1}} \ldots p_{i_{t-1}}^{\gamma_{t-1}} \cdot q_{j_{1}}^{\delta_{1}} \ldots q_{j_{\ell}}^{\delta_{\ell}} \cdot b_{3}, \gamma_{i}, \delta_{i} \geq 1, p^{-}\left(b_{3}\right) \geq p_{s}\right. \\
\left.p \mid b_{3} \Rightarrow p \in \mathbb{P} \backslash Q \text { or } b_{3}=1\right\} .
\end{gathered}
$$


One can write

$$
E^{*}\left(b^{*}\right)=\left\{b \leq n: b=b^{*} b_{3}^{\prime}, b_{3}^{\prime} \in \phi\left(n / b^{*}, T\right)\right\},
$$

where $T$ is the same as in (5.6). Hence

$$
\left|E^{*}\left(b^{*}\right)\right|=\left|\phi\left(n / b^{*}, T\right)\right|=\left|\left(n p_{s} / a^{*}, T\right)\right| .
$$

From the definitions of $E\left(a^{*}\right)$ and $E^{*}\left(b^{*}\right)$ we know that for every $a^{*} \in Z^{1}$ and $b^{*}=a^{*} / p_{s}$,

$$
E_{0}^{*}\left(b^{*}\right)=E\left(a^{*}\right),
$$

where $E_{0}^{*}\left(b^{*}\right)=\left\{b \in E^{*}\left(b^{*}\right): p_{s} \mid b\right\}$, and that (by Lemma 6(2))

$$
\left(E^{*}\left(b^{*}\right) \backslash E_{0}^{*}\left(b^{*}\right)\right) \cap A=\emptyset .
$$

Hence, in the light of (5.5)-(5.10), sufficient for (5.4) is

$$
\left|E^{*}\left(b^{*}\right)\right| \geq 2\left|E\left(a^{*}\right)\right| \quad \text { for every } a^{*} \in Z^{1}, b^{*}=a^{*} / p_{s},
$$

which by (5.7) and (5.8) is equivalent to

$$
\left|\phi\left(n p_{s} / a^{*}, T\right)\right| \geq 2\left|\phi\left(n / a^{*}, T\right)\right|
$$

for $T=\left(\left\{p_{1}, \ldots, p_{s}\right\} \backslash\left\{p_{i_{1}}, \ldots, p_{i_{t}}\right\}\right) \cup\left(\left\{q \in Q: q>p_{s}\right\} \backslash\left\{q_{j_{1}}, \ldots, q_{j_{\ell}}\right\}\right)$, $a^{*}=p_{i_{1}} \ldots p_{i_{t}} q_{j_{1}} \ldots q_{j_{\ell}} ; p_{i_{1}}<p_{i_{2}}<\ldots<p_{i_{t}}=p_{s}<q_{j_{1}}<\ldots<q_{j_{\ell}}, q_{j_{i}} \in Q$.

Now we are in a position to apply Theorem 3 to show the validity of (5.12). The sets $T_{1}, T_{2}, T_{3}$ of primes in Theorem 3 are now

$$
\begin{gathered}
T_{1}=\left\{p_{1}, \ldots, p_{s}\right\} \backslash\left\{p_{i_{1}}, \ldots, p_{i_{t}}\right\} \\
=\left\{p_{1}, \ldots, p_{s-1}\right\} \backslash\left\{p_{i_{1}}, \ldots, p_{i_{t-1}}\right\} \quad\left(p_{i_{t}}=p_{s}\right), \\
T_{2}=\left\{q \in Q: q>p_{s}\right\} \backslash\left\{q_{j_{1}}, \ldots, q_{j_{\ell}}\right\}, \quad \text { and } \quad T_{3}=\left\{p_{i_{1}}, \ldots, p_{i_{t}}\right\} .
\end{gathered}
$$

The condition (3.12), i.e. $u \geq \prod_{p \in T_{2}} p / \prod_{p \in T_{3}} p$, also holds, because $n \geq$ $\prod_{q \in Q} q$ yields

$$
u=\frac{n}{a^{*}}=\frac{n}{p_{i_{1}} \ldots p_{i_{t}} \cdot q_{j_{1}} \ldots q_{j_{\ell}}} \geq \frac{\prod_{q \in Q} q}{p_{i_{1}} \ldots p_{i_{t}} \cdot q_{j_{1}} \ldots q_{j_{\ell}}} \geq \frac{\prod_{q \in T_{2}} q}{\prod_{p \in T_{3}} p} .
$$

This proves (5.12) and consequently (5.3):

$$
\left|A^{1}\right|=\left|M\left(R_{0} \cup \ldots \cup R_{s-1} \cup G_{s}^{1}\right) \cap \mathbb{N}(n)\right| \geq|A| .
$$

Hence $A^{1} \in \mathcal{O}(n, Q)$, because $A \in \mathcal{O}(n, Q)$ and $A^{1} \in I(n, Q)$. Obviously, $P\left(A^{\prime}\right) \subset R_{0} \cup \ldots \cup R_{s-1} \cup G_{s}^{1}$. Therefore $p^{+}\left(P\left(A^{\prime}\right), Q\right)<p_{s}$, which contradicts the definition $p_{s}=\min _{B \in \mathcal{O}(n, Q)} p^{+}(P(B), Q)$. This proves (5.1).

Since for every $B \in \mathcal{O}_{1}(n, Q)$ we have $b_{i} \in P(B)$ it follows that either $p \nmid b_{i}$ for all $p \in \mathbb{P} \backslash Q$ or $2 \mid b_{i}$, but $p \nmid b_{i}, p \in \mathbb{P} \backslash(Q \cup\{2\})$. Let

$$
q_{t}=\min _{B \in \mathcal{O}_{1}(n, Q)} p^{+}(P(B), \phi),
$$


and let

$$
\mathcal{O}_{2}(n, Q)=\left\{B \in \mathcal{O}_{1}(n, Q): p^{+}(P(B), \phi)=q_{t}\right\} .
$$

Again, it is easy to see that $\mathcal{O}_{2}(n, Q) \cap C(Q) \neq \emptyset$. Let $A \in \mathcal{O}_{2}(n, Q) \cap C(Q)$. We write $P(A)$ in the form

$$
P(A)=S_{1} \dot{\cup} \ldots \dot{\cup} S_{t},
$$

where $S_{i}=\left\{b \in p(A): p^{+}(b)=q_{i}\right\}, 1 \leq i \leq t \leq r$.

We are going to prove that $P(A)=\left\{q_{1}\right\}$ if $t=1$, and $P(A)=\left\{2 q_{1}, \ldots\right.$ $\left.\ldots, 2 q_{t}, q_{1} \ldots q_{t}\right\}$ if $t>1$, and this is equivalent to the statement (1.4) of Theorem 1 .

If $t=1$, then clearly $P(A)=\left\{q_{1}\right\}$ and the theorem is true. Hence we assume $t>1$. We observe that $\left\{q_{t}\right\} \notin S_{1}$, because otherwise $\left\{q_{1}\right\} \in S_{1}$ as well, since $A \in C(Q)$ and hence $\left(q_{t}, q_{1}\right)=1$ in contradiction to $A \in I(n, Q)$. Let us assume that

$$
2 q_{t} \notin S_{t} .
$$

Since $A \in \mathcal{O}_{2}(n, Q) \subset \mathcal{O}_{1}(n, Q)$, (5.13) means that every integer $a \in S_{t}$ has at least two different primes from the set $Q$ in its prime decomposition (one of these primes is of course $q_{t}$ ).

Let us prove that the assumption (5.13) is false. We take a similar approach as for proving (5.1). Let $S_{t}=S_{t}^{0} \cup \dot{\cup} S_{t}^{1}$, where $S_{t}^{0}=\left\{a \in S_{t}: q_{t-1} \mid a\right\}$, $S_{t}^{1}=S_{t} \backslash S_{t}^{0}$, and

$$
V_{t}^{i}=\left\{m \in \mathbb{N}: m q_{t} \in S_{t}^{i}\right\}, \quad i=0,1 .
$$

Under assumption (5.13) it can be shown that

$$
A^{i}=M\left(S_{1} \cup \ldots \cup S_{t-1} \cup V_{t}^{i}\right) \cap \mathbb{N}(n) \in I(n, Q), \quad i=0,1 .
$$

Using the approach described in the first part of this section it can be proved that at least one of the inequalities

$$
\left|A^{0}\right| \geq|A|, \quad\left|A^{1}\right| \geq|A|
$$

holds. We mention that only a very special case of Lemma 2 has been used and not Theorem 3 . We also note that here we do not need a restriction on $n$ like $n \geq \prod_{q \in Q} q$.

It can be seen that (5.14) contradicts $A \in \mathcal{O}_{2}(n, Q)$ and hence the assumption (5.13) is false. Therefore $2 q_{t} \in S_{t}$ for $A \in \mathcal{O}_{2}(n, Q) \cap C(Q)$ and $P(A)=S_{1} \cup \ldots \cup S_{t}$.

However, from $2 q_{t} \in S_{t} \subset A \in \mathcal{O}_{2}(n, Q) \cap C(Q)$ it follows that $2 q_{1}, \ldots$ $\ldots, 2 q_{t-1} \in A$ as well and that $q_{i} \notin A$ for all $q_{i} \in Q$. Hence $2 q_{1}, 2 q_{2}, \ldots, 2 q_{t} \in$ $P(A)$.

Let $a \in P(A)$ and $a \neq 2 q_{i}, i=1, \ldots, t$. Since $p^{+}(a) \leq q_{t}\left(A \in \mathcal{O}_{2}(n, Q)\right)$, it follows that $2 \nmid a$ for otherwise $2 q_{i} \mid a$ for some $i \leq t$, which is impossible, 
because $P(A)$ is primitive. Therefore $2 \nmid a$ and $a=q_{1} \ldots q_{t}$, because otherwise $\left(a, 2 q_{i}\right)=1$ for some $i \leq t$. Hence $P(A)=\left\{2 q_{1}, \ldots, 2 q_{t}, q_{1} \ldots q_{t}\right\}$ and Theorem 1 is proved.

Proof of Theorem 2. Since the proof is very similar (and much easier) than the proof of Theorem 1, we will give only a sketch.

We repeat all steps up to formula (5.4) (proof of which was the most difficult part of Theorem 1) and observe that (5.4) trivially holds for squarefree numbers without any restriction on $n$. The situation is similar with formula (5.14) (which was the second main step in the proof of Theorem 1).

6. Example of $Q \subset \mathbb{P}$ and $n<\prod_{q \in Q} q$ for which the conclusion of Theorem 1 does not hold. We take $Q \subset \mathbb{P}$ as follows:

$$
Q=\left\{q_{1}, q_{2}, \ldots, q_{r-1}, q_{r}\right\}=\left\{5,7, \ldots, p_{r+1}, q_{r}\right\},
$$

i.e. $q_{i}=p_{i+2}, i=1,2, \ldots, r-1$, and $q_{r}$ is a prime specified in (6.3) below. We also assume that

$$
q_{r-1}=p_{r+1}>1000
$$

Let

$$
n=2 \cdot 3 \cdot 11 \cdot \prod_{i=1}^{r-1} q_{i}
$$

Finally, for $q_{r} \in \mathbb{P}$ we take any prime satisfying

$$
\frac{n}{2000}<q_{r}<\frac{n}{1000} .
$$

The existence of such primes follows from Bertrand's postulate. We use the abbreviation

$$
H_{j}=M\left\{2 q_{1}, 2 q_{2}, \ldots, 2 q_{j}, q_{1} \ldots q_{j}\right\} \cap \mathbb{N}(n), \quad j=1, \ldots, r .
$$

We are going to prove that for the specified $Q \subset \mathbb{P}$ and $n$, the conclusion of Theorem 1 does not hold, i.e.

$$
f(n, Q)>\max _{1 \leq j \leq r}\left|H_{j}\right| .
$$

We show first that

$$
\max _{1 \leq j \leq r}\left|H_{j}\right|=\max \left\{\left|H_{r-1}\right|,\left|H_{r}\right|\right\} .
$$

Since $2 \prod_{i=1}^{r-1} q_{i} \mid n$, it is easy to see that

$$
\left|H_{j}\right|=n \cdot \frac{1}{2}\left(1-\prod_{i=1}^{j}\left(1-\frac{1}{q_{i}}\right)+\frac{1}{q_{1} \ldots q_{j}}\right) \quad \text { for all } 1 \leq j \leq r-1
$$


and that $\left|H_{2}\right|<\ldots<\left|H_{r-1}\right|$. This proves (6.5), because

$$
\left|H_{1}\right|=\frac{1}{5} n<\frac{1063}{5005} n=\left|H_{4}\right|
$$

and trivially $r-1 \geq 4$ (see (6.1)).

Clearly, to prove (6.4), it is sufficient to find a set $A \in I(n, Q)$ for which

$$
|A|>\max _{1 \leq j \leq r}\left|H_{j}\right|=\max \left\{\left|H_{r-1}\right|,\left|H_{r}\right|\right\} .
$$

We choose $A$ as follows:

$$
A=M\left\{2 q_{1}, 2 q_{2}, \ldots, 2 q_{r-1}, 2 \cdot 3 \cdot q_{r}, 3 \cdot q_{1} \ldots q_{r-1}\right\} \cap \mathbb{N}(n) .
$$

Obviously, $A \in I(n, Q)$ and we have to show that

$$
|A|>\left|H_{r-1}\right|
$$

and

$$
|A|>\left|H_{r}\right|
$$

We consider first the set $H_{r-1} \backslash A$. Since

$$
H_{r-1}=M\left\{2 q_{1}, \ldots, 2 q_{r-1}, q_{1} q_{2} \ldots q_{r-1}\right\} \cap \mathbb{N}(n),
$$

the set $H_{r-1} \backslash A$ consists only of integers of the form

$$
a q_{1} q_{2} \ldots q_{r-1} \leq n=2 \cdot 3 \cdot 11 \cdot q_{1} \ldots q_{r-1}=66 q_{1} \ldots q_{r-1}
$$

and $(a, 6)=1$, because for $(a, 6) \neq 1$ we have $a q_{1} \ldots q_{r-1} \in A$. There are exactly 22 integers $a$ with $a \leq 66$ and $(a, 6)=1$. Hence

$$
\left|H_{r-1} \backslash A\right|=22 \text {. }
$$

Now we consider the set $A \backslash H_{r-1}$. It is clear that all integers of the form $2^{\alpha} \cdot 3^{\beta} \cdot q_{r} \leq n, \alpha \geq 1, \beta \geq 1$, are in $A \backslash H_{r-1}$. We verify that there are 24 integers of the form $2^{\alpha} \cdot 3^{\beta}<1000, \alpha \geq 1, \beta \geq 1$ and since $1000 q_{r}<n$ (by (6.3)) we conclude that $\left|A \backslash H_{r-1}\right| \geq 24>\left|H_{r-1} \backslash A\right|=22$. This proves (6.6).

To prove (6.7) we compare the cardinalities of $H_{r} \backslash A$ and $A \backslash H_{r}$. Since $H_{r}=M\left\{2 q_{1}, 2 q_{2}, \ldots, 2 q_{r-1}, 2 q_{r}, q_{1} \ldots q_{r}\right\} \cap \mathbb{N}(n)=M\left\{2 q_{1}, \ldots, 2 q_{r}\right\} \cap \mathbb{N}(n)$ (because $q_{1} \ldots q_{r}>n$ ), $H_{r} \backslash A$ consists only of integers of the form

$$
2 \cdot q_{r} \cdot b \leq n
$$

where $b$ is not divisible by any of the primes $3, q_{1}, \ldots, q_{r-1}$. Since $q_{r}>$ $n / 2000$ (see (6.3)), we conclude from (6.8) that $b<1000$. However, since $q_{r-1}>1000$ (see (6.1)), we have $b \in\left\{1,2, \ldots, 2^{9}\right\}$ and hence

$$
\left|H_{r} \backslash A\right| \leq 10 \text {. }
$$

Now we consider the set $A \backslash H_{r}$. It consists of the integers of the form

$$
3 \cdot q_{1} \ldots q_{r-1} \cdot c \leq n=66 q_{1} \ldots q_{r-1},
$$


where $2 \nmid c$. There are exactly 11 such integers $c \leq 22$. Hence

$$
\left|A \backslash H_{r}\right|=11>10=\left|H_{r} \backslash A\right|
$$

and this proves (6.7).

7. Directions of research. We think that our methods are applicable to other number theoretical extremal problems.

A first question is how $f(n, Q)$ can be characterized if $Q$ is an infinite set of primes. Perhaps more demanding is the problem of finding a common generalisation of the problem analysed in this paper and its in dual in [1]:

For (finite) sets $Q_{1}, Q_{2} \subset \mathbb{P}, Q_{1} \cap Q_{2}=\emptyset$, and $n \in \mathbb{N}$, what is the maximal cardinality $k$ of sets $A=\left\{a_{1}<\ldots<a_{k}\right\} \subset \mathbb{N}(n)$ satisfying $\left(a_{i}, a_{j}\right) \neq 1$, $\left(a_{i}, \prod_{q \in Q_{1}} q\right) \neq 1$, and $\left(a_{i}, \prod_{q \in Q_{2}} q\right)=1$ for all $i, j$ ?

Instead of requiring that no two numbers of $A$ are relatively prime one can require that no $\ell$ numbers are pairwise relatively prime.

\section{References}

[1] R. Ahlswede and L. H. Khachatrian, Sets of integers and quasi-integers with pairwise common divisor, Acta Arith. 74 (1996), 141-153.

[2] - - - Maximal sets of numbers not containing $k+1$ pairwise coprime integers, ibid. 72 (1995), $77-100$

[3] P. Erdős, Problems and results in combinatorial number theory, Chapt. 12 in: A Survey of Combinatorial Theory, J. N. Srivastava et al. (eds.), North-Holland, 1973.

[4] -, A survey of problems in combinatorial number theory, Ann. Discrete Math. 6 (1980), 89-115.

[5] P. Ribenboim, The Little Book of Big Primes, Springer, 1991.

[6] J. B. Rosser and L. Schoenfeld, Approximate formulas for some functions of prime numbers, Illinois J. Math. 6 (1962), 64-89.

Universität Bielefeld

Fakultät für Mathematik

Postfach 100131

33501 Bielefeld, Germany

E-mail: lk@mathematik.uni.bielefeld.de 